01,04

\title{
Автогенерация хаотического СВЧ-сигнала в спин-волновом оптоэлектронном генераторе
}

\author{
() А.Б. Устинов, А.В. Кондрашов, А.А. Никитин, А.В. Дроздовский, Б.А. Калиникос \\ Санкт-Петербургский государственный электротехнический университет „ЛЭТИ“, \\ Санкт-Петербург, Россия \\ E-mail: ustinov-rus@mail.ru
}

\begin{abstract}
Впервые экспериментально исследована автогенерация хаотического сверхвысокочастотного (СВЧ) сигнала в спин-волновом оптоэлектронном генераторе. Генератор представлял собой кольцевую схему, в СВЧ-тракте которой находились спин-волновая линия задержки на пленке железо-иттриевого граната (ЖИГ) и СВЧ-усилитель. Оптический тракт содержал оптоволоконную линию задержки. Нелинейным элементом генератора являлась пленка ЖИГ, работавшая в условиях четырехволнового параметрического взаимодействия спиновых волн. Показано, что, управляя коэффициентом усиления кольца, можно генерировать монохроматический, периодический, квазипериодический и хаотический СВЧ-сигналы. Определены параметры, характеризующие генерируемый кольцом хаотический сигнал.
\end{abstract}

Экспериментальная часть работы выполнена при частичной поддержке Российского научного фонда (грант № 16-12-10440). Теоретическая часть работы выполнена при поддержке госзадания Минобрнауки РФ.

DOI: $10.21883 /$ FTT.2018.11.46643.20NN

\section{1. Введение}

Спиновые волны (СВ), распространяющиеся в ферромагнитных пленках на сверхвысоких частотах (СВЧ), демонстрируют богатую нелинейную динамику. Солитоны огибающей и модуляционная неустойчивость СВ стали уже привычными нелинейными эффектами, наблюдаемыми при распространении интенсивных СВ. Для экспериментов с нелинейными спиновыми волнами обычно выбирают монокристаллические пленки железо-иттриевого граната (ЖИГ). Эти пленки получили широкое распространение благодаря рекордно низким потерям на распространение $\mathrm{CB}$, порядка $20 \mathrm{~dB} / \mu \mathrm{s}$, a также низкому порогу возникновения нелинейных эффектов порядка десятков милливатт.

Если с выхода спин-волновой линии задержки, изготовленной на пленке ЖИГ, взять СВЧ-сигнал, усилить его и опять подать на вход, то такая схема будет представлять собой активный кольцевой резонатор. В случае полной компенсации потерь в активном кольце можно генерировать различные виды сигналов: монохроматический $[1]$, в виде периодической последовательности нелинейных импульсов - солитонов огибающей СВ [2] и сигналы в форме динамического хаоса [3]. Последние два режима генерации реализуются в кольце за счет возникновения и развития нелинейных эффектов при распространении СВ в ферромагнитной пленке.

Исследование возможностей генерации СВЧ динамического хаоса и перехода к хаосу в различных нелинейных автоколебательных системах представляет значительный интерес, который обусловлен возможностью прикладного использования динамического хаоса. Так, динамический хаос можно использовать для построения телекоммуникационных систем, георадаров и систем генерации случайных чисел [4-6]. Недавно было про- демонстрировано использование динамического хаоса в системах радиоосвещения - освещения предметов и поверхностей искусственными некогерентными источниками микроволнового излучения с целью их последующего наблюдения при помощи радиометрической аппаратуры [7].

Спин-волновые генераторы хаоса на пленках ЖИГ можно разделить на две группы. К первой группе относятся генераторы, в основе работы которых лежат трехволновые процессы параметрического взаимодействия СВ $[8,9]$. Такие процессы для пленок ЖИГ разрешены в нижней части СВЧ-диапазона на частотах до $4.9 \mathrm{GHz}$. Выше этой частоты трехволновые процессы запрещены законами сохранения энергии и импульса и нелинейная динамика СВ определяется четырехволновыми процессами. Поэтому ко второй группе относятся генераторы хаоса, в которых с ростом СВЧ-мощности развиваются четырехволновые параметрические процессы $[10,11]$. Исследование генерируемых спин-волновым генератором параметров непериодических сигналов подтверждает динамическую природу хаотического сигнала, а также позволяет выявить возможность управления его характеристиками для реализации управляемого генератора СВЧ динамического хаоса.

В последние годы повышенный интерес приобрели также исследования оптоэлектронных генераторов сверхвысокочастотного хаоса [12-15], сконструированных с использованием принципов радиофотоники [16]. Такие радиофотонные генераторы изготавливаются по кольцевой схеме с последовательно соединенными оптическим и радиочастотным трактами. В оптическом тракте осуществляется задержка СВЧ-сигнала, а в радиочастотном тракте - его усиление. Нелинейным элементом таких генераторов является электрооптический модулятор типа Маха-Цендера, который модулирует 
амплитуду оптического излучения СВЧ-сигналом из радиочастотного тракта.

Целью настоящей работы являлось исследование автогенерации солитонов огибающей и динамического хаоса в спин-волновом оптоэлектронном СВЧ-генераторе, сконструированном в виде активного резонансного кольца, нелинейная динамика которого определяется нелинейностью спиновых волн, распространяющихся в пленке ЖИГ. Исследования проводились на частотах, где трехволновые процессы параметрического взаимодействия СВ были запрещены законами сохранения, а нелинейная динамика $\mathrm{CB}$ определялась только четырехволновыми процессами.

\section{2. Экспериментальный макет}

Экспериментальный макет генератора был построен по кольцевой схеме, которая состояла из радиочастотного и оптического трактов (рис. 1). Оптический тракт состоял из лазерного модуля $l$ с длиной волны оптического излучения $1.55 \mu \mathrm{m}$, электрооптического модулятора типа Маха-Цендера 2, оптоволоконного кабеля 3 длиной $100 \mathrm{~m}$ и фотодетектора 4. Рабочая точка модулятора была смещена в квадратуру для обеспечения максимальной линейности преобразования СВЧ-сигнала в оптический.

Радиочастотный тракт содержал СВЧ-усилители 5, переменный аттанюатор 6, спин-волновую линию задержки 7 на монокристаллической пленке ЖИГ и направленный ответвитель 8 . Пленка ЖИГ была эпитаксиально выращена на подложке из галлий-гадолиниевого граната. Длина пленки была $20 \mathrm{~mm}$, толщина $3.1 \mu \mathrm{m}$. Пленка была намагничена поперечно, что позволяло возбуждать в ней поверхностные спиновые волны. Напряженность магнитного поля составляла 1200 Ое. В качестве элементов возбуждения и приема спиновых волн использовались входная и выходная микрополосковые антенны шириной $50 \mu \mathrm{m}$. Расстояние между антеннами составляло $3 \mathrm{~mm}$.

Рассмотрим принцип работы генератора. Лазерный диод 1 излучает непрерывное оптическое излучение, выполняющее функцию несущего сигнала. Электрооптический модулятор 2 модулирует амплитуду оптического излучения СВЧ-сигналом, поступающим из радиочастотного тракта. Модулированное оптическое излучение, распространяясь по оптоволоконному кабелю 3, задерживается в нем и детектируется фотодетектором 4. Полученный сверхвысокочастотный сигнал усиливается с помощью первого СВЧ-усилителя 5 и, пройдя аттенюатор 6, поступает на вход спин-волновой линии задержки 7. Сигнал с выхода линии задержки усиливается с помощью второго СВЧ-усилителя 5 и подается на электрооптический модулятор 2, замыкая кольцевую схему. Пара СВЧ-усилителей 5 компенсирует суммарные потери на распространение СВЧ-сигнала в кольце. Переменный аттенюатор 6 служит для управ-

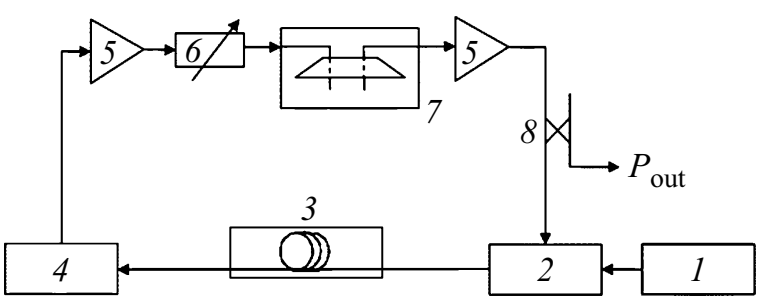

Pис. 1. Блок-схема экспериментального макета: 1 - лазерный модуль; 2 - электрооптический модулятор; 3 - оптоволоконный кабель; $4-$ фотодетектор; 5 - СВЧ-усилитель; $6-$ переменный аттенюатор; 7 - спин-волновая линия задержки; 8 - направленный ответвитель.

ления этими потерями. Небольшая часть СВЧ-сигнала выводится из кольца направленным ответвителем 8 с коэффициентом связи $-10 \mathrm{~dB}$.

В описанной конструкции нелинейные свойства осциллятора определяются нелинейными свойствами спиновых волн, распространяющихся в пленке ЖИГ. Как уже говорилось, порог возникновения спин-волновых параметрических нелинейных явлений составляет десятки милливатт, что значительно меньше порогов возникновения нелинейных явлений в элементах оптического тракта. Усилители СВЧ-сигнала при таких уровнях мощности также работают в линейном режиме.

Отметим, что спин-волновую линию задержки можно использовать в качестве элемента, задающего частоту полосы хаотической генерации. Эту полосу можно перестраивать по частоте, изменяя поле подмагничивания пленки ЖИГ.

\section{3. Экспериментальные результаты}

Экспериментальное исследование перехода к автогенерации хаотического сигнала проводилось путем плавного увеличения значения динамического параметра - коэффициента усиления кольца $G$. Увеличение $G$ осуществлялось за счет уменьшения ослабления СВЧ-сигнала в переменном аттенюаторе 6. Значение $G$, при котором кольцо переходило в режим автогенерации, было условно принято равным нулю. Таким образом, значение $G=0$ соответствовало порогу автогенерации СВЧ-сигнала. Увеличение коэффициента усиления кольца приводило к последовательной смене режимов автогенерации, обуславливаемой возникновением нелинейных четырехволновых параметрических процессов в пленке ЖИГ. Рассмотрим эти режимы.

На рис. 2 представлены частотные спектры мощности и временные профили амплитуды огибающей СВЧ-сигнала, генерируемого в кольце. При некотором значении ослабления в кольце возникла монохроматическая генерация СВЧ-сигнала. Как уже говорилось, это значение коэффициента усиления $G$ было условно принято равным нулю. Частота генерируемой гармоники со- 

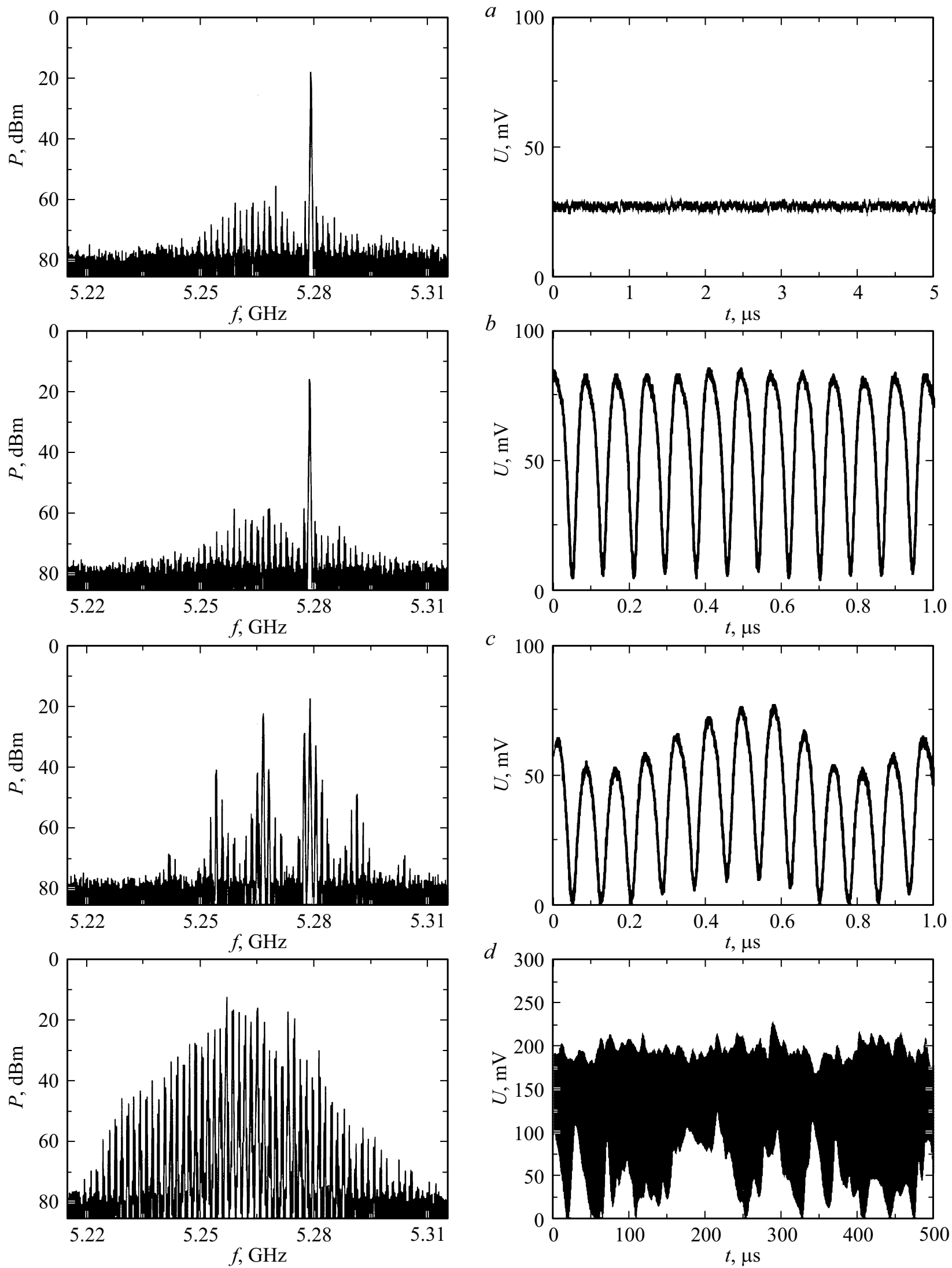

Рис. 2. Спектры (слева) и осциллограммы (справа) СВЧ-сигналов, генерируемых при $G=0 \mathrm{~dB}(a), G=0.05 \mathrm{~dB}(b)$ и $G=0.4 \mathrm{~dB}(c), G=1.29 \mathrm{~dB}(d)$. 


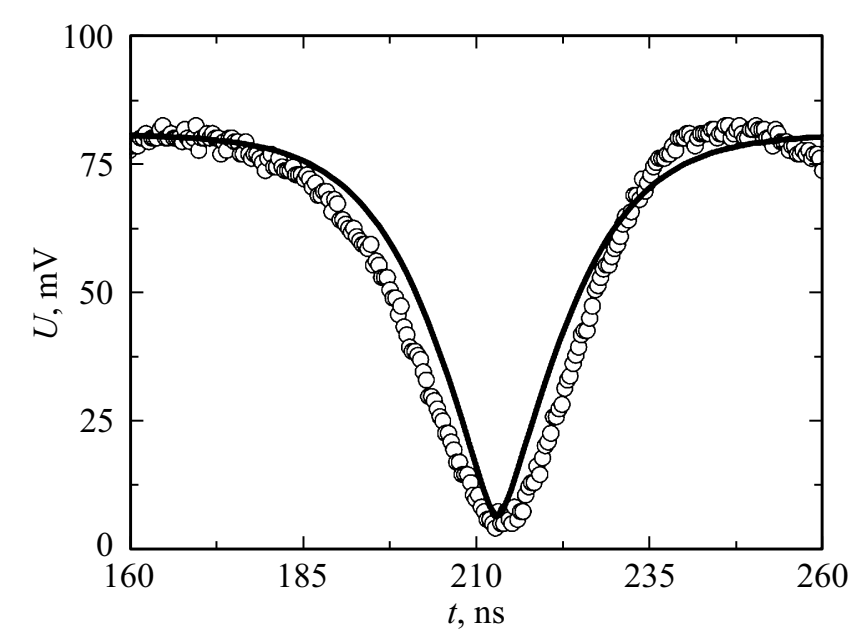

Рис. 3. Экспериментальный (кружки) и теоретический (линия) профили темного солитона.

ставляла $5.2788 \mathrm{GHz}$. Во временной области наблюдался постоянный уровень огибающей генерируемого сигнала. При увеличении коэффициента усиления $G$ до $0.05 \mathrm{~dB}$ мощность СВЧ-сигнала на входе спин-волновой линии задержки начинала превышать порог возникновения нелинейного четырехволнового параметрического взаимодействия поверхностных спиновых волн. При этом автогенерация монохроматического СВЧ-сигнала сменялась многочастотной автогенерацией. Во временной области наблюдалась автогенерация последовательности темных нелинейных импульсов - солитонов огибающей спиновых волн. Период следования импульсов составил $80.5 \mathrm{~ns}$, а их длительность - примерно $22.8 \mathrm{~ns}$.

Поскольку нелинейная теория оптоэлектронных СВЧ-генераторов отсутствует, солитонная природа импульсов подтверждалась путем моделирования формы отдельных импульсов с помощью формулы, описывающей решение нелинейного уравнения Шредингера для темных солитонов (см., например, [17]). Так как оптический тракт работал в линейном режиме, то нелинейный коэффициент $N$ и дисперсионный коэффициент $D$ были рассчитаны из дисперсионной характеристики поверхностных спиновых волн. В качестве подгоночного параметра была использована групповая скорость спиновых волн. Оказалось, что расчетная форма солитона находится в хорошем соответствии с полученной экспериментально. Сопоставление экспериментального и расчетного амплитудных профилей показано на рис. 3.

Генерация солитоноподобных импульсов наблюдалась до $G=0.4 \mathrm{~dB}$. При превышении этого значения в спектре генерации появлялись новые гармоники, как показано на рис. 2,c. При этом периодическая последовательность солитонов приобретала амплитудную модуляцию. При достижении коэффициентом усиления $G$ значения $1.29 \mathrm{~dB}$, стационарная автогенерация сменилась режимом генерации хаотического сигнала. При этом в спектральной области наблюдаюсь формиро- вание сплошного спектра. Ширина полосы генерации составляла примерно $100 \mathrm{MHz}$. Во временной области такому спектру соответствовал непериодический сигнал. Дальнейшее увеличение $G$ не приводило к заметным изменениям ни в спектральной, ни во временной областях вплоть до значения $G=3 \mathrm{~dB}$.

\section{4. Анализ режимов генерации}

Для анализа природы наблюдавшихся режимов автогенерации по измеренным временным реализациям были построены фазовые портреты, соответствующие различным режимам работы генератора. В режиме генерации монохроматического сигнала и последовательности темных солитонов фазовые портреты представляли собой точку и замкнутую линию. Это свидетельствует о том, что в этих режимах в фазовом пространстве последовательно сменяли друг друга регулярные аттракторы. Монохроматическому режиму соответствовал аттрактор в форме устойчивой точки, периодическому режиму - предельный цикл. При увеличении коэффициента усиления $G$ до $0.4 \mathrm{~dB}$ возникновение вторичной модуляционной неустойчивости приводило к разрушению предельного цикла и формированию фазового портрета в виде двумерного тора. В пользу этого вывода говорит также то, что спектр сигнала содержит гармоники с частотами, находящимися в иррациональном соотношении. При последующем увеличении тор разрушался, фазовый портрет принимал сложную форму, что во временной области соответствовало переходу к генерации непериодического СВЧ-сигнала. На рис. 4 показана двумерная проекция фазового портрета, соответствующая $G=1.29 \mathrm{~dB}$. Последующее увеличение коэффициента усиления кольца не приводило к значительным изменениям фазового портрета. Описанный сценарий перехода к генерации динамического хаоса соответствует сценарию Рюэля-Такенса.

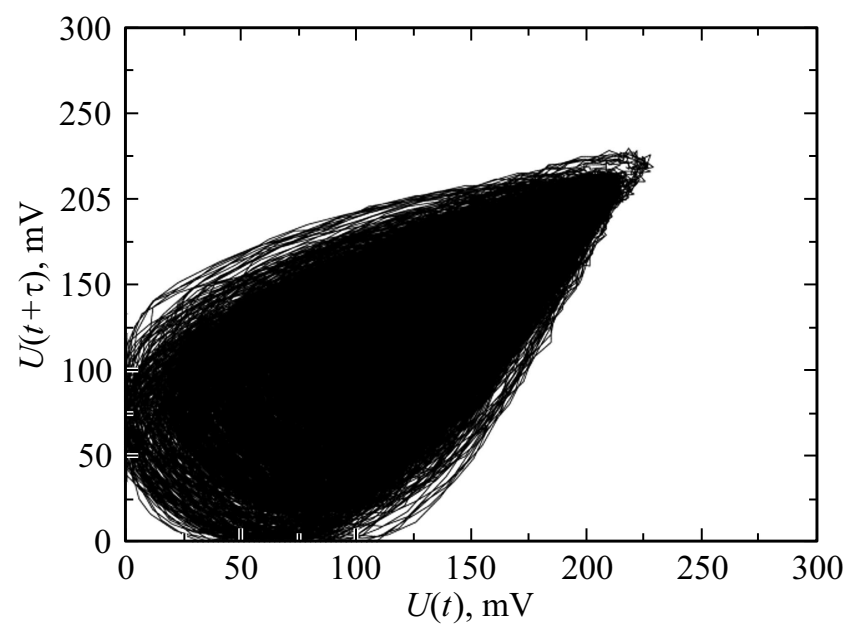

Рис. 4. Двумерный фазовый портрет СВЧ-сигнала в режиме хаотической генерации для $G=1.29 \mathrm{~dB}$. 
Для подтверждения сделанных предположений о сценарии перехода к хаосу и о динамической природе хаотических колебаний была проведена оценка топологии аттракторов по построенным фазовым портретам. Были рассчитаны значения фрактальной размерности, используя алгоритм Грассбергера-Прокаччиа. Расчет показал, что в режиме генерации монохроматического сигнала фрактальная размерность была близка к нулю, что характерно для аттракторов в форме устойчивой точки. При переходе к генерации последовательности солитонов фрактальная размерность скачком увеличивалась до единицы, что соответствует предельному циклу. При переходе к генерации модулированной последовательности солитонов фрактальная размерность возрастала до значения, равного 2. Переход к генерации динамического хаоса характеризовался скачком фрактальной размерности до величины 2.5 при $G=1.29 \mathrm{~dB}$. Полученные значения фрактальной размерности подтверждают вывод о сценарии перехода от стационарной генерации к генерации динамического хаоса.

Дополнительно были рассчитаны значения старшего показателя Ляпунова. Полученные результаты показали, что в режиме монохроматической генерации старший показатель Ляпунова был отрицательным, а в периодических режимах он равнялся нулю. В режиме генерации динамического хаоса старший показатель Ляпунова имел положительное значение.

\section{5. Заключение}

Из проведенного исследования следует, что кольцевые оптоэлектронные СВЧ-генераторы, использующие в радиочастотном тракте в качестве нелинейного элемента спин-волновую линию задержки на пленке ЖИГ, могут генерировать как стационарный, так и хаотический сигнал. Изменяя величину поля подмагничивания, возможно выбирать частоту генерируемого сигнала. Управляя коэффициентом усиления кольца, можно изменять вид генерации.

В отличие от работы [14], где нелинейность оптоэлектронного генератора обусловлена нелинейным коэффициентом передачи электрооптического модулятора типа Маха-Цендера, в настоящей работе названный модулятор работал в линейном режиме, а нелинейные свойства генератора определялись нелинейностью пленки ЖИГ. Величина магнитного поля влияла на порог автогенерации в соответствии с изменением эффективности возбуждения спиновых волн и их групповой скорости. Изменение величины поля не меняло качественную картину перехода к хаосу при сохранении условия работы модулятора Маха-Цендера в линейном режиме. Таким образом, спин-волновые оптоэлектронные СВЧ-генераторы могут найти практическое применение как электрически управляемые генераторы СВЧ-сигналов.

\section{Список литературы}

[1] A.V. Drozdovskii, A.B. Ustinov. J. Phys.: Conf. Ser. 661, 012062 (2015).

[2] M.M. Scott, B.A. Kalinikos, C.E. Patton. Appl. Phys. Lett. 78, 970 (2001).

[3] А.В. Кондрашов, А.Б. Устинов, Б.А. Калиникос, Н. Benner. Письма в ЖТФ 34, 81 (2008).

[4] А.А. Короновский, О.И. Москаленко, А.Е. Храмов. УФН 179, 1281 (2009).

[5] Chaotic Signal Processing / Ed. H. Leung. Higher Education Press: Beijing (2014). 189 p.

[6] W. Li, I. Reidler, Y. Aviad, Y. Huang, H. Song, Y. Zhang, M. Rosenbluh, I. Kanter. Phys. Rev. Lett. 111, 044102 (2013).

[7] А.С. Дмитриев, Е.В. Ефремова. Письма в ЖТФ 42, 49 (2016).

[8] В.Е. Демидов, Н.Г. Ковшиков. ЖТФ 69, 100 (1999).

[9] С.В. Гришин, В.С. Гришин, Д.В. Романенко, Ю.П. Шараевский. Письма в ЖТФ 40, 51 (2014).

[10] A. Hagerstrom, M. Wu, R. Eykholt, B.A. Kalinikos. Phys. Rev. B 83, 104402 (2011).

[11] А.В. Кондрашов, А.Б. Устинов, Б.А. Калиникос. Письма в ЖТФ 42, 82 (2016)

[12] L. Larger, J.M. Dudley. Nature 465, 41 (2010).

[13] K.E. Callan, L. Illing, Z. Gao, D.J. Gauthier, E. Scholl. Phys. Rev. Lett. 104, 113901 (2010).

[14] А.В. Кондрашов, А.Б. Устинов, Б.А. Калиникос. ЖТФ 87, 259 (2017).

[15] Y.K. Chembo. Opt. Lett. 42, 3431 (2017).

[16] М.Е. Белкин, А.С. Сигов. Радиотехника и электроника 54, 901 (2009).

[17] M. Remoissenet. Waves Called Solitons: Concepts and Experiments. Springer-Verlag, Berlin (1999). 328 p.

Редактор Т.Н. Василевская 